\title{
Jnhalt
}

Seite

Einleit $\mathfrak{u} \mathfrak{n} \boldsymbol{8}$

1. Erfenntnistheoretilde Borerinnerung . . . . . . 1

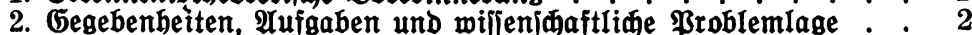

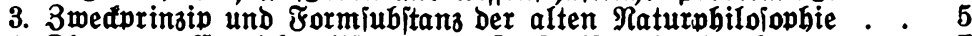

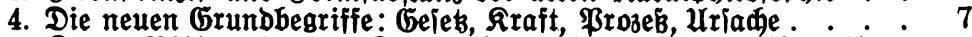

5. Rants Philoiophie Des Drganilimen und bie Raturphiloiophie Der IDealilten

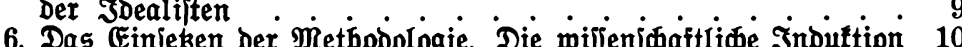

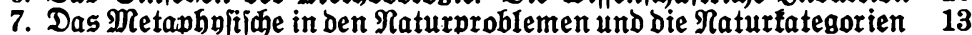

8. Die Bearbeitbarfeit unlösbarer $\mathfrak{P r o b l e m e}$. . . . . . . 15

9. Rlaififife und moderne $\mathfrak{B h y}$ fit. Relativismus und Spielraum Der Raturphiloiophie . . . . 17

10. Ermeitertes Gefintsfeld. Rosmologiine ßeripettinen. . . . 18

11. Die Grenzen bes Mathematifihen in ben Gubitraten Der Grö̈e . 20

12. Rategoriale Stellung Des Mathematilden in ben $\mathfrak{R a t u r =} 22$ phänomenen

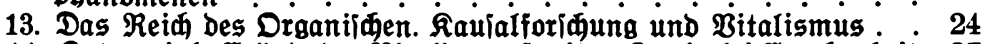

14. Rategoriale Grïnde bes Bitalismusitreites. 3weierlei Gregebenbeit 27

15. Rategoriale Grenzïberidreitung von zwei Seiten her.... 29

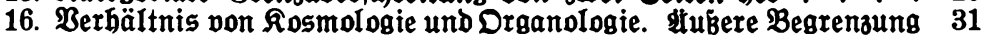

17. Beiondere $\mathfrak{A} u f g a b e n ~ u n d ~ i n n e r e ~ G r e n z e ~ b e r ~ \mathfrak{A n a l y j e ~ . ~ . ~ . ~ . ~ . ~} 33$

18. Die erfenntnistheoretiide Geite bes Rategorienproblems . . . 35

19. 3ur Methodenlehre ber Rategorialanalyje . . . . . . . 38

\section{Eriter Teil}

\section{Dímenfionale Rategoríen}

\section{21bidnitt. Dimenfionen Der realen Belt}

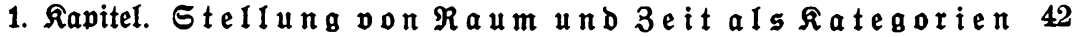

a. $\mathfrak{B o m} \mathfrak{A}$ nfang Der $\mathfrak{R a t u r v h i l o i o p h i e ~ . . . . . . . . . ~} 42$

b. Die Dimenfionsfategorien und bie bejonberen Dimenfionen. . 44

c. Inhaltsleere Dimenitonalität

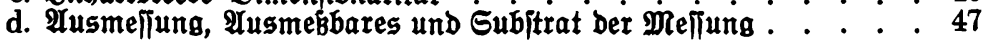

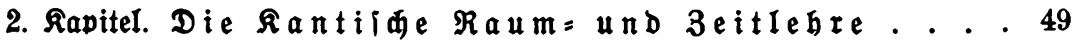

a. Innifaungsformen und Rategorien . . . . . . . . . . 49

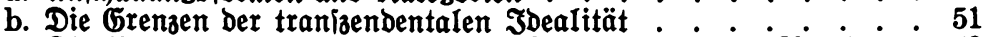

c. Die Berboppelung Der $\mathfrak{R a u m}=$ und Beitanalpie. Reue $\mathfrak{A p d r i e n ~}$. 53 


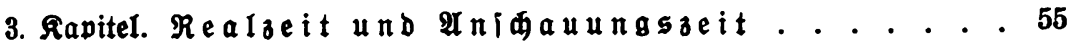

a. Bemubtiein ber Zeitliđfeit und 3eitliđłteit bes Bewubtieins . . 55

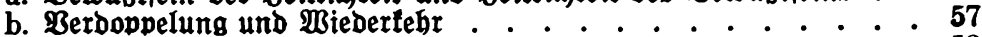

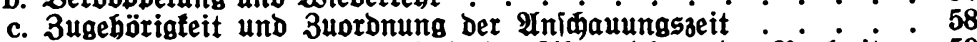

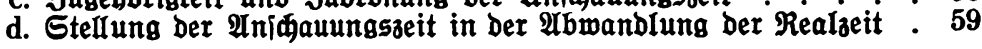

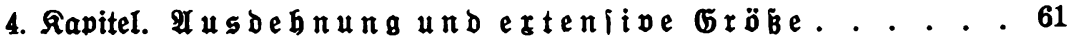

a. Extenfion und Dimenfion . . . . . . . . . . . . 61

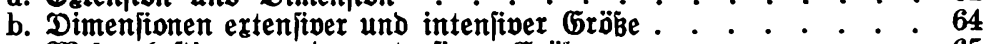

c. Weiensbejtimmung der ertenfiven Gröbe . . . . . . . . . 65

d. Die Kategorien $\mathfrak{D}$ Lá und Gröвe . . . . . . . . . . . . 67

\section{II. :Gbifnitt. Rategorialanalyie Des Ranmes}

5. Rapitel. Der ge ometriføe $\Re \mathfrak{a} u \mathfrak{m}$. . . . . . . . . . . 69

a. Das Ineinanbergreifen Der Probleme . . . . . . . . . . 69

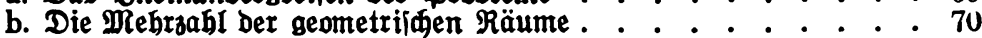

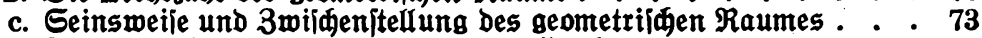

d. Die eriten fategorialen Momente bes ibealen Raumes . . . . . 75

e. Weitere tategoriale Pomente Des idealen Raumes . . . . . . 77

f. Folgerungen aus Den getroffenen Bejtimmungen . . . . . . 79

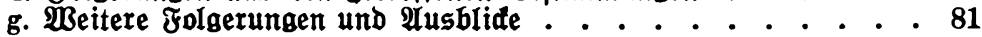

6. Kavitel. Der $\mathfrak{R} \mathfrak{a} \mathfrak{l} \mathfrak{r} \mathfrak{a} \mathfrak{u} \mathfrak{m}$. . . . . . . . . . . . . 83

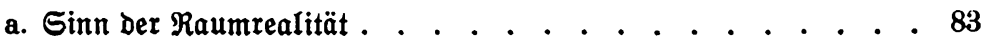

b. Einzigfeit, Dimenfionenzahi und Gubjtratøaratter des Realraumes 85

c. Der Realraum als reines Dimenfionsiyjtem . . . . . . . . 89

d. Somogeneität, Stetigteit und Unbegrenatheit bes Realraumes . 92

e. Das an fí Gröbenloje . . . . . . . . . . . . . . . 95

f. Das in fid Mabloje . . . . . . . . . . . . . . . . . 96

g. Das Eentreळtitehen ber Dimenfionen aufeinanber und bie empirifaen Roordinatenipiteme . . . . . . 98

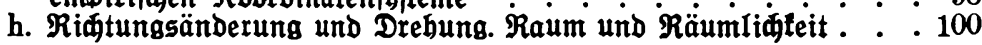

7. Rapitel. Di e $\Re$ äumlidte it ber Dinge . . . . . . . . 103

a. Das Unerfennbare im Realraume . . . . . . . . . . . . 103

b. Gründe gegen bas receptaculum rerum . $: .0_{0}: .104$

c. Die Relativismen ber Realräumliđłe it (Größe, \&age, Riðtung) . 107

d. Die Relativismen Der Bewegung . . . . . 108

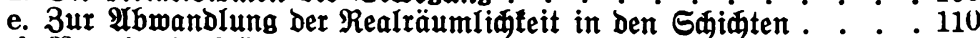

f. Bermittelte Räumliøłeit in Den höheren Seinsiałaten . . . . 112

8. Savitel. Der $\mathfrak{A} \mathfrak{n} \mathfrak{i} \mathfrak{a} \mathfrak{a} \mathfrak{u} \mathfrak{n} \mathfrak{g} \mathfrak{s} \mathfrak{r} \mathfrak{a} \mathfrak{u}$. . . . . . . . . . . . 113

a. Wabrneদmungsraum, Erlebnisraum u. a. m. . . . . . . . . 113

b. Tajtraum und Sebraum. Ertreme Theorien . . . . . . . 114

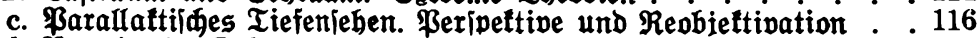

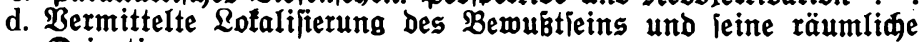
Orientierung . . . . . . . . . . . 118

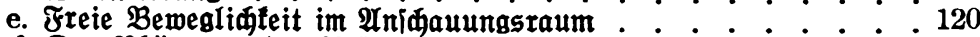

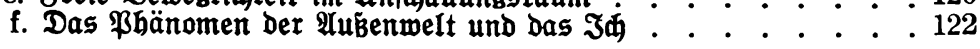

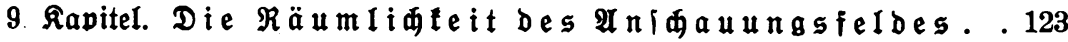

a. Die mit bem Realraume gemeiniamen Momente . . . . . . . 123

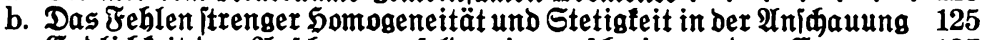

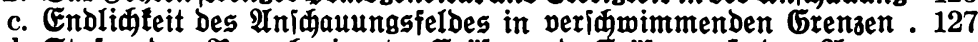

d. Stufen ber Raumborizonte. Gröвe und Eröвenmaß bes $\mathfrak{A} n=$ iđauungsraumes

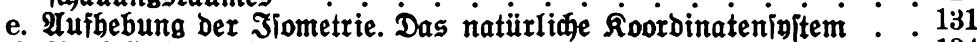

f. Beiđränfte Geltung bes receptaculum rerum . . . . . . 134 


\section{Abiñnitt. Sategorialanalyle bet Beit}

10. Gapitel. Problemanlä be ber 3 eitanalyie..... . 136

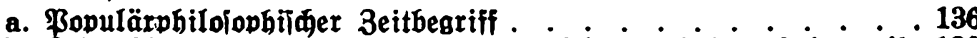

b. Zeitliđłteit und Realität. Bergängliđ̆leit als höhere Seinsmeile 138

c. Das ßroblem Der ibealen 3eit . . . . . . . . . . . . . 140

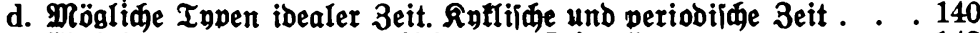

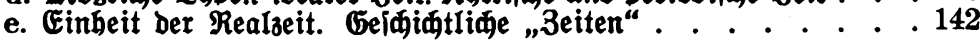

11. Rapitel. Die Realze it . . . . . . . . . . . . . 144

a. Sinn ber Beitrealität . . . . . . . . . . . . . . 144

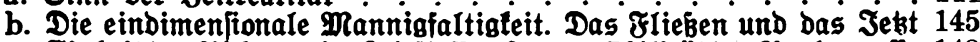

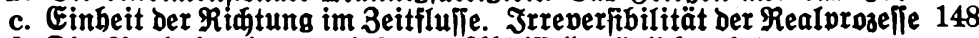

d. Die Realzeit als gemeiniame "İbizilfe möglider beterogener Drbinaten . . . . . . . . . . . 151

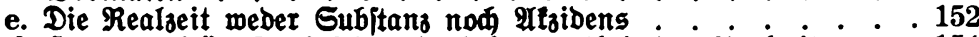

f. Somogeneität, Stetigleit und unbegrenztheit Der Realseit . . . 154

g. Realzeit als bas an fí Gröbenlole und Máblole . . . . . 158

12. Rapitel. Die 3 eitlidfeit ber Realvrozelle.... . 161

a. Das Unerfennbare in ber Realzeit . . . . . . . . . . . 161

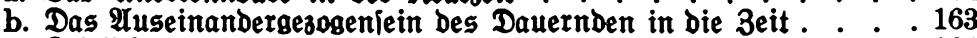

c. Beitliđes Beilammeniein und Áseinanderjein . . . . . . . 164

d. 3eitmobi eriter Drbnung. Gegenmart und Bergangenbeit . . . . 165

e. Seinsweile bes Zufünftigen. Das unaufhaltiam Înrïđende: . . 167

13. Rapitel. Die 3 eitmodi höberer Drbnung . . . . . 170

a. 3eitmodi zweiter Dronung. Die Simultaneität. . . . . . . 170

b. Die Gufzelfion und íre Ridtung. . . . . . . . . . . . 172

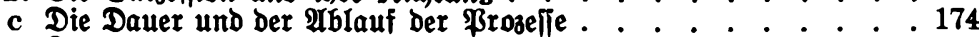

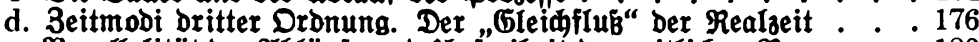

e. Farallelität Der $\mathfrak{A b l a ̈ u f e ~ u n o ~ U n f r e i h e i t ~ b e r ~ s e i t l i đ e n ~ B e w e g u n g ~ . ~} 180$

f. Das Fehlen zeitliđer Eigenbewegung. Realseitlide Determination 182

g. Das Borrüđen Des Jebt und Die Erhaltung im Jebt. . . . . 185

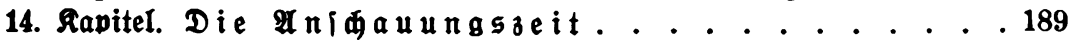

a. Abmandung Der 3eitfategorie . . . . . . . . . . . . . 189

b. æahrnebmungszeit, Erlebnisseit und Borltellungszeit : . . 190

c. Das Erleben und bie Erhaltung Des erlebenden Subjeftes ..$\quad 192$

d. Die freie Bemegliđfeit bes voritellenben Subielts in ber $\mathfrak{A} n=$ iळauungszeit . . . . . . . . . . . . . . 194

e. Die verbreiterte Bemubtieinsgegenmart.. .5196

f. Die 3eitperipettive und Der Borgriff auf bie 3ufunft . . . . . 197

g. Das J̌fthalten bes Bergangenen. Erinnerung unb Erfabrung: $: 200$

15. Rapitel. Die 3 eitlid

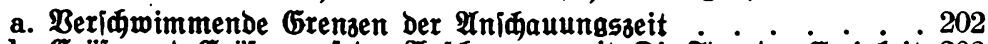

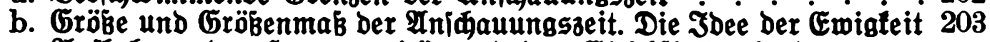

c. $\mathfrak{T}$ ufhebung ber gomogeneität und bes Gleiđflulles in ber Inidaung . . . . . 206

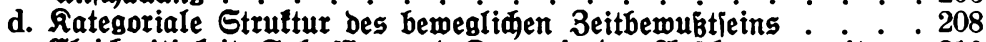

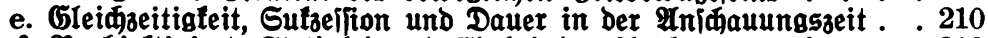

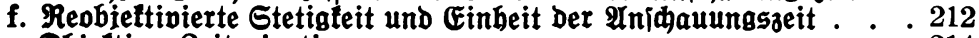

g. Dbieftive Zeitorientierung . . . . . . . . . . . 214

\section{Abidnitt. Das Ranms3eitipltem Dex Ratur}

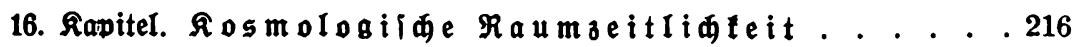

a. Das vierbimenfionale Snitem . . . . . . . . . . . . 216

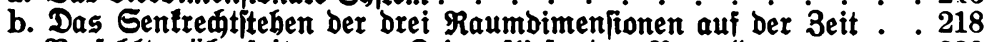

c. Berfeblte Itberipibungen. Rein "fließender Raum". . . . 220 
d. Unaufhebbarfeit bes beterogenen Subitratøarafters in Raum und 3eit

e. Bezogenbeit beterogener Dimenfionsinjteme auf bie Cinbeit Der Realbeit ................... . . 224

17. Rapitel. Die $\{a t e g$ orie ber $\mathfrak{B}$ ewegung. . . . . . . 226

a. Bewegung als Urphänomen Der Raumseitlidłteit . . . . . 226

b. Die fategorialen Momente ber Bewegung . . . . . . . . . 228

c. Berbältnis Der Raum= und Beitmomente in ber Bemegung . .230

d. Die einfade Relativität ber Bewegung im vierbimenftonalen Snitem

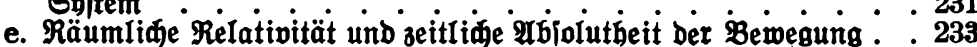

18. Rapitel. Spefulative Relativismen bes $R a \mathfrak{l}$ mes

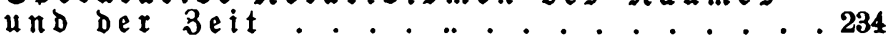

a. Die ßroblem/ituation Der Relatinitätstheorie . . . . . . . . 234

b. Die Relativierung Der Gleiđzeitigleit . . . . . . . . . 236

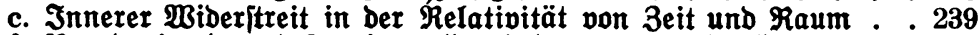

d. Paraborien bes "Deformierten“ und Des "rotierenben“ Raumes ${ }^{242}$

e. Liđ̆tgeiđwwinbigleit als poitulierte Ronitante aller Relativität . 245

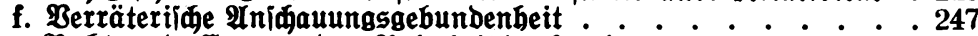

g. Ređ̆t und Grenzen ber Relativitätstheorie . . . . . . . . 249

\section{3 weiter Teil}

\section{RKosmologí(the RKategotíen}

\section{Abinnitt. Das Berben und Die Beharrung}

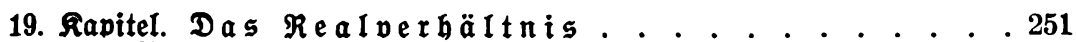

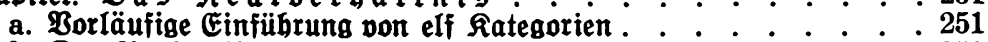

b. Das Realverhältnis als Die innere Relationalitä̀t ber Dinge . 253

c. Das Realverhältnis zmiiðen Beitimmtheiten veriđiebener

Dimenitonen

d. Das Realverhältnis als Bemuktieinstategorie : . . . . . 257

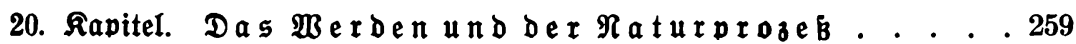

a. Das zeitliđe Sein bes Werbens . . . . . . . . . . . . . 259

b. Sijtorijळes. Sein und Berben, Entítehen und Bergeben . $: .260$

c. Beränberung und Bemegung. Beweglides Realverhältnis . . . 262

d. Relativität Der Bewegung und Яbiolutheit Der Beränderung : 264

21. Ravitel. Modalanalyie bes Prozelfes...... . . . 265

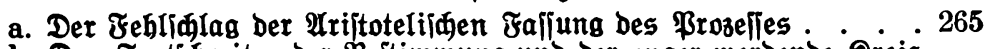

b. Das Fortiđreiten Der Beitimmung und Der enger werbende Rreis

Des Mögliđen . . . . . . • . . . . . . . 267

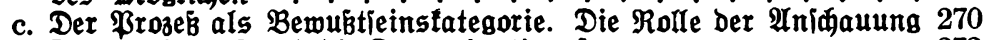

d. Der Realproseb und die Determinationsformen . . . . . . 272

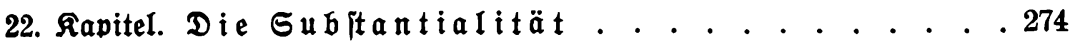

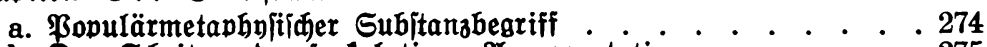

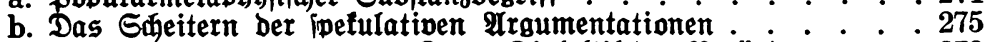

c. Die Subitanz als Materie. Fants Dialeftił Der Beränderung . 278

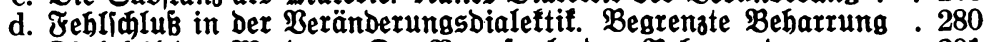

e. Dialeftif bes Werbens. Der $\mathfrak{B r o z e b}$ als das Bebarrende. . . . 281 
23. Rapitel. Die Bebarrang und bas Bebarrende. . . 284

a. Das Miberitehen im Prozeb als Beharren im Jebt. . . . 284

b. Synthele von Subltrat und Bebarruns. Das Quantitative in ber Subitanz

c. Rritif trabitioneller Subitanapräbifate. Relativität ber Subjtanz 289

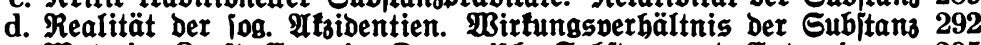

e. Paterie, Rraft, Energie. Dnnamilae Subitans und Entrovie . . 295

f. Das Bureiđen relativ beharrender Subjtrate im ßeltproseß : . 297

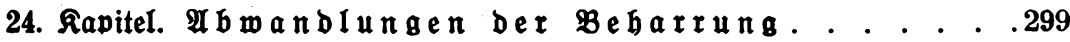

a. Beharrung obne Subitrat und Gubititenz . . . . . . . . . 299

b. Beharrung und Erhaltung. Subfiłtenz, Trägheit und Ronjiłtens .301

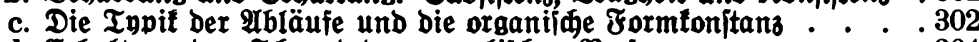

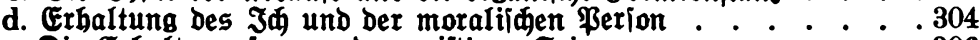

e. Die Erhaltungsformen Des geiftigen Geins. . . . . . . . . . .306

f. Die Subitanz als Bemubtieinsfategorie . . . . . . . . . . .308

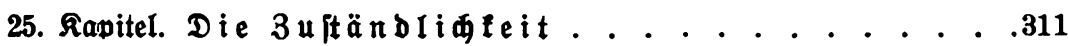

a. Gubitanる und 3ujtand . . . . . . . . . . . . . . . .311

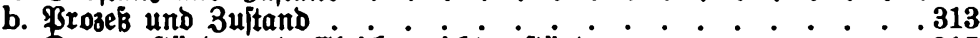

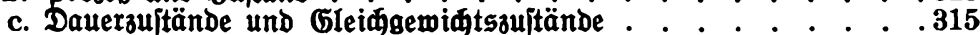

d. Die 3uitändiððleit als Bewustieinstategorie . . . . . . . .316

\section{Abiduitt. Die Ranialität}

26. Rapitel. Die taulale Determinationsform . . . .318

a. 3eitliđe Drbnungsfolge und $\mathfrak{A b h a ̈ n g i g f e i t ~ . ~ . ~ . ~ . ~ . ~ . ~ . ~ . ~ . ~} 318$

b. Das Raulalgeies und die Realnotwendigleit ber Raulalfolge : . 321

c. Die Raulalreibe, Der RaulaInerus und ber Raulalproseb. . . 323

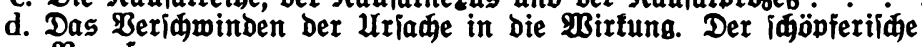
Proseb

e. Die Unertennbarteit bes Gervorbringens . . . . . . . 328

27. Ravitel. $3 \mathfrak{u} \mathfrak{x}$ Metapbyif ber Beruriadung . . . . . 331

a. Raulalverbältnis und Finalverbältnis . . . . . . . . . . .331

b. Rategoriale juberformbarteit bes Raulalnerus . . . . . . . . 333

c. Pluralität Der Teilmögliðłteiten und Einheit Der Realmögliđ̆leit . 334

d. Der Ginn ber Raujalnotwendigteit. Grenzen Der Unabwenbbarłeit . 337

e. Das Berhältnis ber ßaulalität zur Gubitanz . . . . . . . . 339

f. Die fortlaufend veriळobene Jontität von Uriaße unb Wirtung . 341

28. Rapitel. Romplezes Bemirten und Einmaligleit.. .343

a. Irrationalität ber wirfliđđen causa efficiens . . . . . . . . . 343

b. Der iđeinbare modus deficiens und ber affirmative Charafter aller Raulalfattoren .

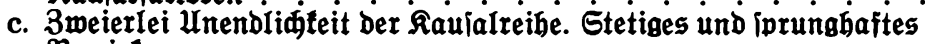
Bewirfen . . . . . . . . . . . .

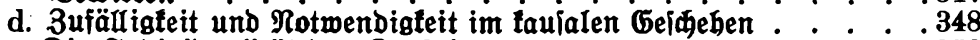

e. Die Jnbinibualität bes Raulalnerus . . . . . . . . . 350

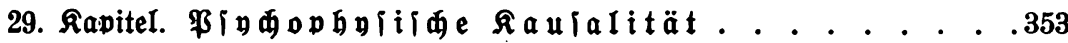

a. श̂bmandlungen bes Bewirtens . . . . . . . . . . . . 353

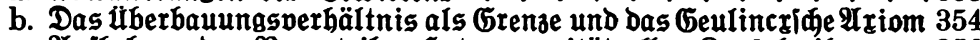

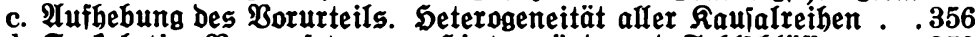

d. Spefulative Borausiebungen, Sintergriunde und Jehlihlïlle. .359

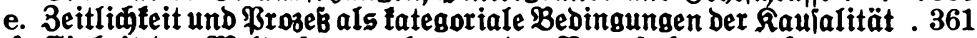

f. Einheit Des Beltzulammenhanges ber Beruriađung naळ. . . . 363 
30. Rapitel. Die $\mathfrak{A} u \mathfrak{f}$ eisbarleit bes Raufaloufamens

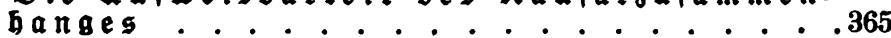

a. Das $\mathfrak{B r o b l e m}$ ber obieftiven Gültigleit . . . . . . . . . .365

b. Der mobale Beweisgang und feine Fehler. $\because 366$

c. Gume und Rant. "(Eewöbnung" und "Innalogie ber Erfabrung". 368

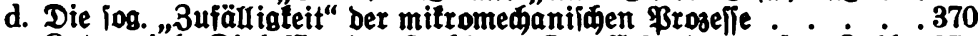

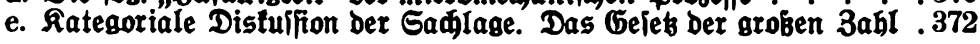

31. Rapitel. Raulalität als Bewubtieinstategorie . . 376

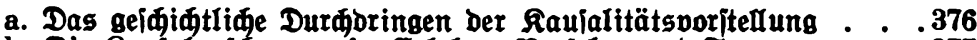

b. Die Ћaulalanidauung im Erleben, Boriehen und Iun . . . . . 377

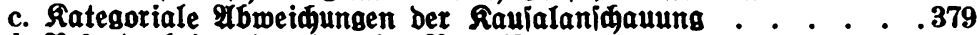

d. Beionderbeiten bes taulalen Begreifens . . . . . . . . . .380

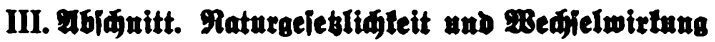

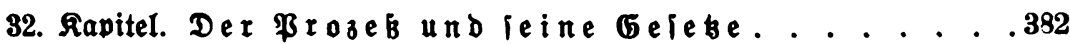

a. Die Frormentppif Der $\mathfrak{A b l a ̈ u f e ~ . ~ . ~ . ~ . ~ . ~ . ~ . ~ . ~ . ~ . ~ . 3 8 2 ~}$

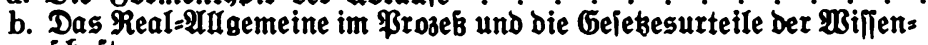

iffaft

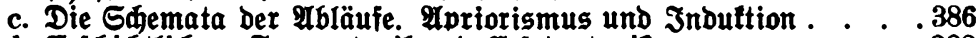

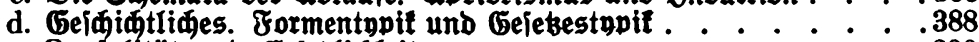

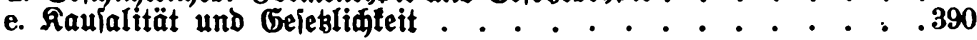

33. Rapitel. Das $\mathfrak{R} a \mathfrak{t}$ rgeies und ieine matbematifide

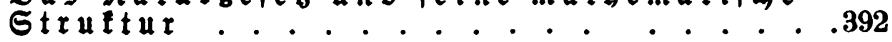

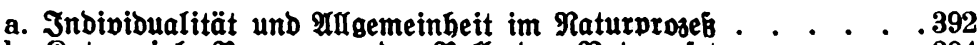

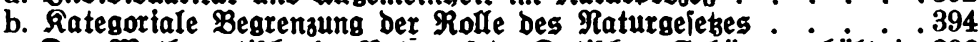

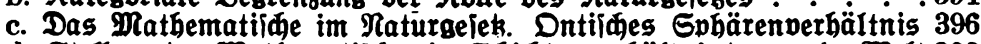

d. Stellung Des Mathematijøen im Sđidtenverhältnis ber realen Welt 398

e. Das Geheimnis ber eratten $\mathfrak{B i}$ ifienidaft . . . . . . . . . .399

34. Rapitel. RIafiifide und itatiftifde Geieblidteit. . 401

a. Die \&unttion. Mathematiiøe Form und Realität . . . . 401

b. Das Continuum Der Bewegung und Das Jnfinitefimalprinzip . 402

c. Die Bereinfađung und ber bypothetilihe Cinidiag in Der Geleses: wi ilenimaft

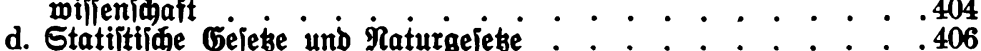

e. Regellofigłeit und Éejebmäßigłeit. Das Ronvergenzpbä̈nomen . .408

35. Rapitel. Raturgeieblidteitals Erenntnistategorie 411

a. Irberipannung des Geiebesgedantens in ber Willenidaft . . . .411

b. Grenzen Des Apriorismus. Ertreme Der Theorie ....413

c. Gejeł und Iatiađe. Berwiurfelung ber Sphären . . .414

d. $\mathfrak{A b l e b n u n g ~ r e l a t i v i t i t i d e r ~ S t e p l i s . ~ I n z e i d e n ~ o b j e t t i p e r ~ G u ̈ i l t i g t e i t ~} 416$

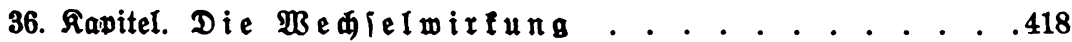

a. Die geididtlide $\mathfrak{B r o b l e m l a g e ~ . . . . . . . . . . ~} .418$

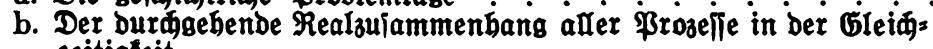
seitigleit . . . . . 419

c. Die Determinationsform der gegenieitigen Bebingtheit : . .421

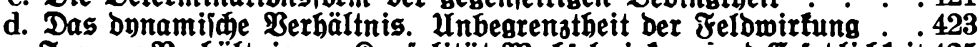

e. Inneres $\mathfrak{B e r h a ̈ l t n i s ~ v o n ~ R a u l a l i t a ̈ t , ~} 2$ Bediel wirtung und Gejebliđiteit 425

f. Gelettive Eunttion Der ßBéfielwirtung . . . . . . . 427 


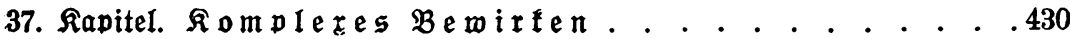

a. Meøanijđe Wirfung und Gegenmirfung . . . . . . . . . .430

b. Das ïberfaulale Moment im fompleren Bewirfen . . . . . . 432

c. Das Wirfungsgefüge in ber Frlühtiglett Der Zuitände : $: .433$

d. Die Form ber Dependent in ber Weblelwirtung. Bierdimentionale Ủ̧̧̋̈ngigfeit . . . . . . . . . . . . . . . . . . .435

e. Wedielwirfung als Bemubtieinstategorie . . . . . .437

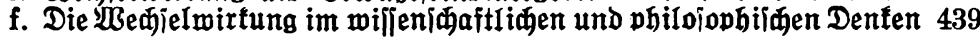

\section{Ibidnitt. Ratïrlidje Gefïge und Eleidgewidte}

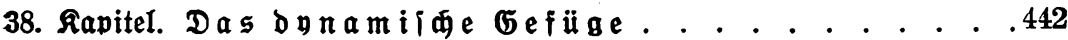

a. Die bistreten Gebilde . . . . . . . . . .442

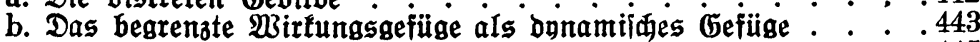

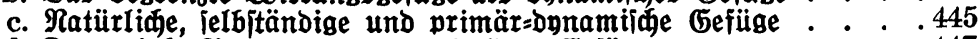

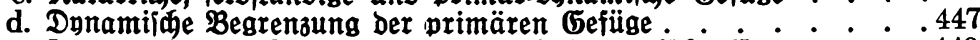

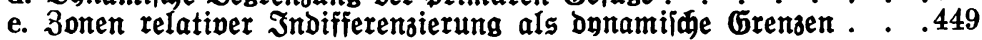

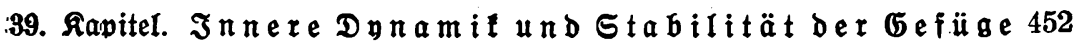

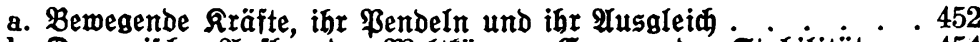

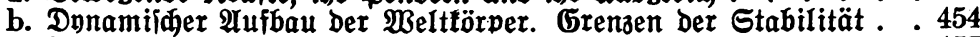

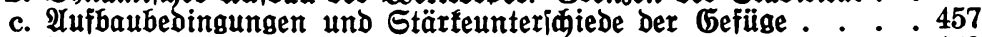

d. Berhältnis von Gröвenoronung und Stärfe ber Gefüge . . . . 458

e. Gijtorifhes. Beiondere Enormen, Geieke und Gebilde . . . . 460

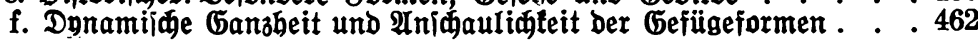

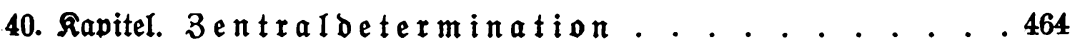

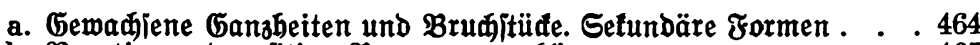

b. Regative und pofitive Begrenzungsphänomene . . . . . . . 465

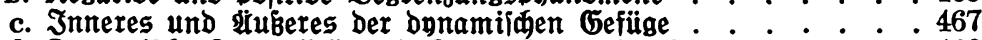

d. Dinamijas Zentralität und Zentralbetermination . . . . . . 468

e. Bewubtieinsfrembheit bes Dynamijđen Gefüges. . . . . . . . 471

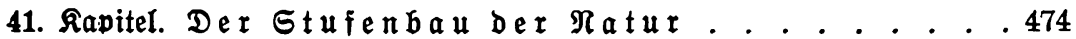

a. Gefüge als Elemente von Gefügen . . . . . . . . . . . . 474

b. Materiefunftion und Gliebfunftion ber Elemente.. .476

c. überformung und $\mathfrak{A} u$ tonomie. Innenträfte und $\mathfrak{A} u$ Benträfte . . 478

d. Das Dnnamilide Grunbgeies bes Stufenbaus . . . . . . . . 480

e. Spaltung Der Dronungsfolge im Etufenbau : $: .0_{0}: .482$

f. Die Rüde im Stufenbau. Stellung ber böditen Gefüge . . . 483

42. Rapitel. Dynami 更e Ganzbeitsbetermination . . 486

a. Recttläufige und rüaläufige Determination . . . . . . . . 486

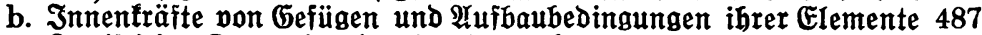

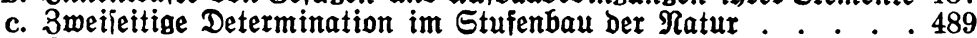

d. Roniequenzen. Gefundäre Ganzheiten und bie log. "Dinge“. . 491

e. $\mathfrak{U}$ bwandlungen und Beionderungen . . . . . . . . . . 493

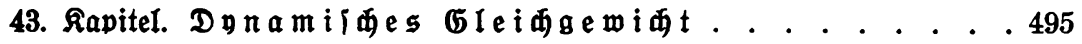

a. Berbältnis von Stabilität und Rabilität in Den Gefügen . . . 495

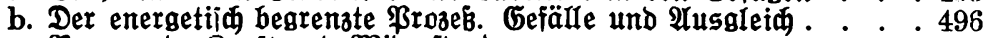

c. Bewegende Rraft und Wiberitand . . . 498

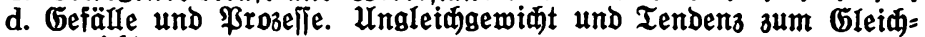
gemicht . . . . . . . . . . . . . . . . . . . 500

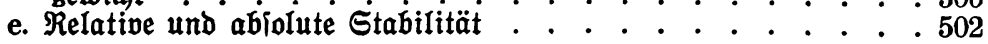


44. Rapitel. Geleftivität ber Gleíggemídte.... . . 504

a. Gefüge und $\mathfrak{X b l a u f s t n p e n ~ . ~ . ~ . ~ . ~ . ~ . ~ . ~ . ~} 504$

b. Dnnamilăe Eeleftinität Der Gefüge und Prozelle . . . . . . 505

c. Sđ̆einbar teleologi đae Berbältnille . . . . . . . . . 507

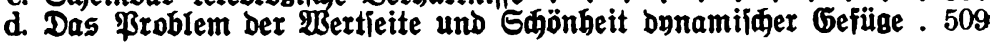

\section{Dritter Ieil}

\section{Organologí(d)e Rategoríen}

\section{27bidnitt. Das organiline Gefüge}

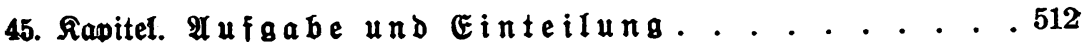

a. Problemlage und $\mathscr{I n}$ Inüpfung . . . . . . . . . . . . . 512

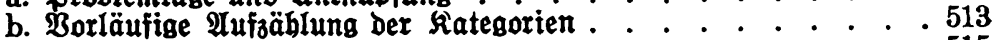

c. Der Drganismus als Jndivibuum . . . . . . . . . . 515

46. Ravitel. Das $\mathfrak{J} \mathfrak{n} \mathfrak{i} \mathfrak{v} \mathfrak{i} \mathfrak{u} \mathfrak{u m}$. . . . . . . . . . . . 516

a. Drganismus und Drgane. Formen und Prozelfe . . . . . . 516

b. Înfang und Ende bes Jnbivibuums. Reben und Tod . . . 518

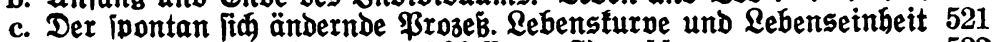

d. Einbeit und Ganzheit Der geidlofienen \$rozebform . . . . . . 522

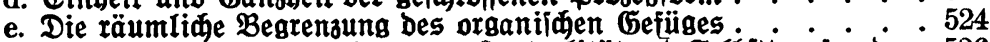

f. Qebensiphäre des Jndidiðuums. Bentralität unb Selbittranizendenz 526

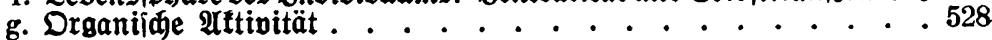

47. Ravitel. Der formbildende $\mathfrak{B}$ rozeb. . . . . . . 530

a. Das Berbältnis von Stoff, Form und Prozeß . . . . . . . 530

b. Bewegliđer Stoff und beweglide form. Der $\mathfrak{B r i m a t}$ bes $\mathfrak{P r o z e l l e s ~} 531$

c. Der ielbittätis aufbauende \$rozes und bas lía felbit erbauende Gefüge

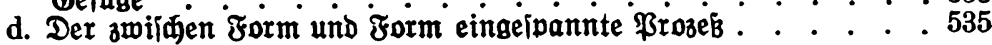

48. Rapitel. Das $\mathfrak{B}$ ideripiel ber $\mathfrak{P r o z e l f e . ~ . ~ . ~ . ~ . ~ . ~} 537$

a. Die fin ielbjt verbraudende und erneuernde Form . . . . . 537

b. Ôfímilation und Difímilation . . . . . . . . . . . . . . 539

c. Die morphogenetiløe $\mathfrak{A}$ ftivität des \$lasmas . . . . . . . . . 541

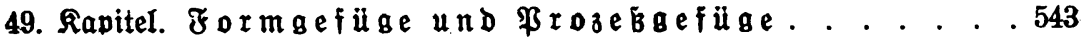

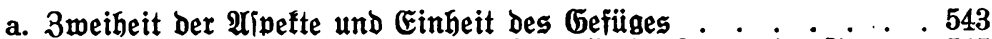

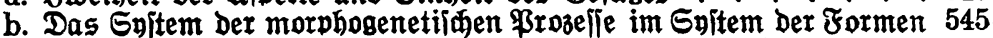

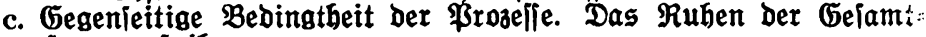
form auf ínen

d. Ronfiltenz als Erhaltungsmobus Der Iebendigen frorm . . . 548

50. Rapitel. Die organifde Gelbitregulation . . . . . 551

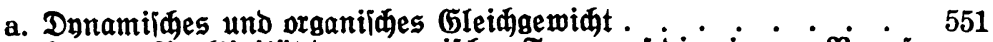

b. Intere Reaftivität ber organilðen Form auf bie eigenen \$rozes=

fomponenten . . . . . . . . . . . . . . • . . 552

c. $\mathfrak{B h a ̈ n o m e n e ~ u n d ~ S i n t e r g r u ̈ n d e . ~ S e l b j t b e g r e n z u n g ~ b e r ~ R e g u l a t i o n ~} 554$

d. 何erprobuftion und Wađstum . • . .

e. Gelbitbegrenzung Des Waぬstums. Regulation böberer Dronung 558 


\section{II. П6)}

51. Rapitel. Das $\mathfrak{L} \mathfrak{e} \mathfrak{b} \mathfrak{n}$ ber $\mathfrak{A} \mathfrak{r}$. . . . . . . . . . 560

a. Die zweite Rategoriengruppe ...........560

b. Die Realität bes Rebens ber $\mathfrak{Y r t}$. . . . . . . . . . 561

c. Einheit und Erhaltung Des überindivibuellen Qebens. . . . 564

d. Das Rebensgefüige böherer Dronung. Stellung bes Indivibuums $\mathfrak{i n}$ ifm

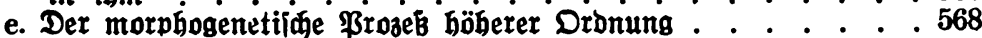

f. Gelbittranizendenz bes Snbivibuums und Ronifiten Des Geiamt= lebens . . . . . . . . 571

g. Das tategoriale Reproduttionsgeiés . . . . . . . . . 573

52. Rapitel. Die Wieberbilbung bes $\mathfrak{J} \mathfrak{b i v i b u}$ ms. . . 575

a. Das Rätiel ber formübertragung . . . . . . . . . . 575

b. Entwid̄lung und Inlageinitem

c. 3enteilung, ,Eene" und proipettive Bebeutung . : . : 579

d. 3ur Determinationsform Des Entwiälungsproselies. . . . 580

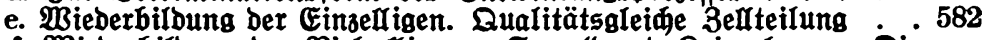

f. Mieberbilbung ber Bielzelligen. "Eoma“ und Keimplasma. Die Bererbung . . . . . . . . . . . . 584

53. אapiteI. I

a. "Potentielle $\mathfrak{U n}_{\text {niterblidfeit" }}$. . . . . . . . . . 586

b. Die Rontinuität bes Reimplasmas. Der abgejpaltene Rebensprozes 587

c. Geidledtliđe Fortpflanzung. Rebuftionsteilung und Befrudtung 589

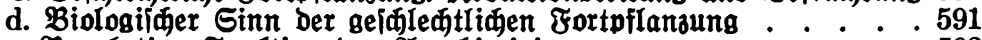

e. Regulative Eunftion ber $\mathfrak{A m p h i m i x i s ~ . ~ . ~ . ~ . ~ . ~ . ~ . ~ . ~} 593$

f. Inzumt und Barthenogeneie. . . . . . . . . . . 594

54. Rapitel. Die $\mathfrak{B a r} \mathfrak{a} \mathfrak{b} \mathfrak{i} \mathfrak{i} \mathfrak{t a ̈ t}$. . . . . . . . . . .596

a. Ratürliđes Snitem und Bewegliøfeit ber $\mathfrak{A r t e n}$. . . . . 596

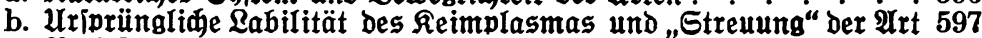

c. Berfejtigung und iefundäre Stabilität . . ...... . 599

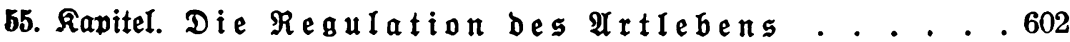

a. Das Iebende Individuum groß̉en Gtils . . . . . . . . 602

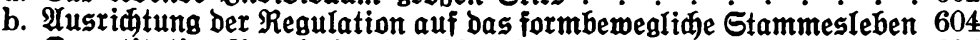

c. Quantitative Regulation Des $\mathfrak{A r t l}$ rebens . . . . . . . . . 605

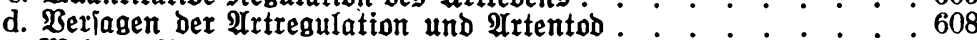

e. Weitere Regulationsformen . . . . . . . . . . 610

\section{A6idnitt. Die $\mathfrak{\text { Phylogeneie }}$}

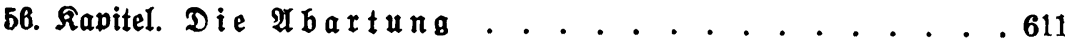

a. Das Sditúal einer Entbedung . . . . . . . . . . . . 611

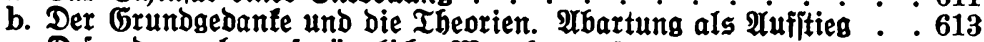

c. Deizendenz als uriprïngliḑe Morphogeneie . . . . . 615

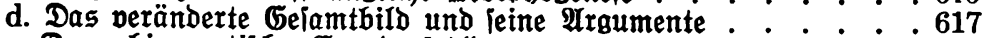

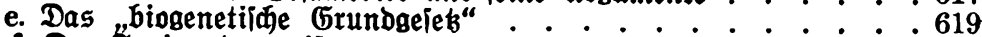

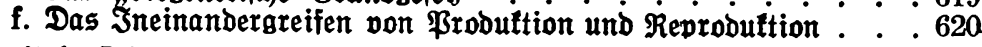

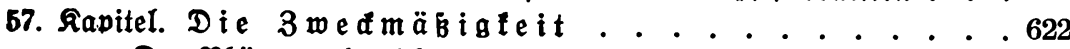

a. Der P̧änomenbereid und bas $\mathfrak{P r o b l e m ~ . ~ . ~ . ~ . ~ . ~ . ~ . ~ . 6 2 2 ~}$

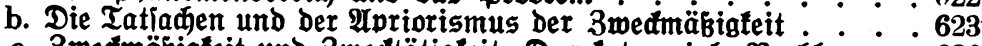

c. ZwedmäBigfeit und 3weđtätigfeit. Das fategoriale Prob̆lem : .626

d. Relative, zufällige und organiiđe 3weé̛mäßigteit . . . . 627

e. Die Theorien. Teleologilihe Errtlärungsweife. . : : : 629

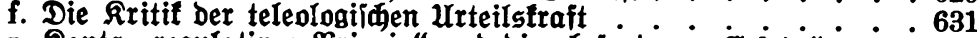

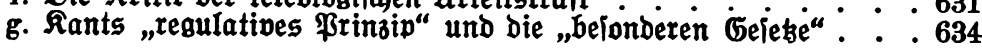


58. Ravitel. Drganiføe Gelettion. . . . . . . . . . . 637

a. Das Problem. Tajtende $\mathfrak{L o ̈ j u n g s p e r j u d e ~ . ~ . ~ . ~ . ~ . ~ . ~ . ~ . ~ 6 3 7 ~}$

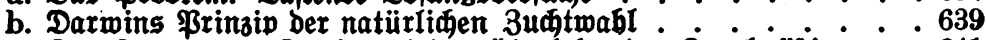

c. Der Ћampf ums Dalein und Das Juberleben Des 3wedmäbigen . . 641

d. Rategorialer Çarafter und $\mathfrak{A}$ priorität bes Seleftionsprinzips . . 643

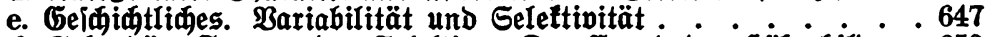

f. Sefunbäre Formen Der Seleftion. Der Grund Der Söberbil bung 650

g. Selettion und Inlagedetermination ............652

59. Rapitel. Die $\mathfrak{M} u t a t i o n$. . . . . . . . . . . . . 655

a. Grenzen ber leleftiven Erłlärung . . . . . . . . . . . 655

b. Bebingungen ber $\mathbb{M}$ utation und $\mathfrak{M} u$ tationsperioden...$\quad .657$

c. Gegenieitiges Berhältnis von Mutation unb Seleftion . . . .660

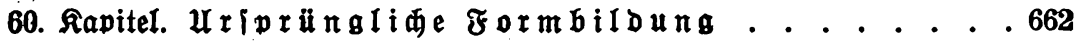

a. Îbitammung und Iuffitieg ber Jormen . . . . . . ... . . 662

b. Die iog. „Jurzeugung" und die fortgeiebt eritmalige Porpbogeneie 664

c. Înfänge und Jortgang ber formenmannigfaltigleit . . . . 665

\section{Ibidnitt. Drganijøe Determination}

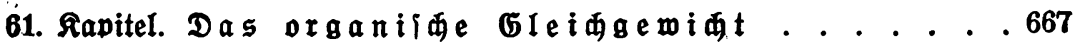

a. Labilität und Stabilität lebender Ganzheiten . . . . . . . 667

b. Erbaltung und İmbildung. Das Durwbređen ber uriprüngliden Labilität ................ . . . 669

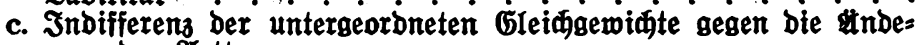
rung bes $\mathfrak{A r t t y p u s} .0670$

d. Die Rolle Des Ungleidgemidts in Der Staffelung ber Ġieid= geminte ................. . . . 672

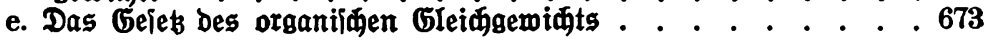

62. Rapitel. Der Lebensprozé . . . . . . . . . . . 675

a. Subjtantialifierung ber Rebendigfeit . . . . . . . . .675

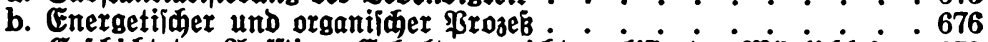

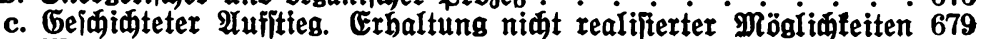

d. Modaler $\mathfrak{B a u}$ ber Dntogenele. Multipotentialität und Regeneration 681

e. Das Problem ber Jntralelettion ............683

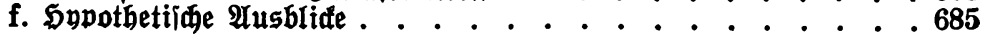

63. Rapitel. Der nexus organicus . . . . . . . . . . . . 687

a. Die falide Irternative ber Iheorien. . . . . . . . . .687

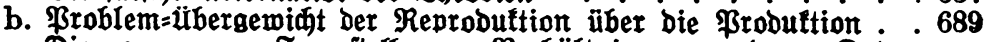

c. Die genauerer Eragejtellung. Berbältnis ou anbern Deters minationsformen . . . . . . . . . . . . . . 691

d. Drganiløe Zentraldetermination. Der geidiolene Uriadientompler 694

e. Die zwectmäßige $\mathfrak{U}$ usleie ber $\mathfrak{U}$ riađenmomente im Reimplasma .696

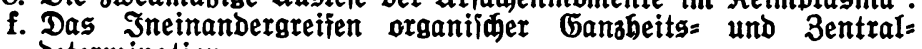
Determination . . . . . . . . 700

g. überiðiळtung und Gefilge ber Determinationen . . . . . . . 702

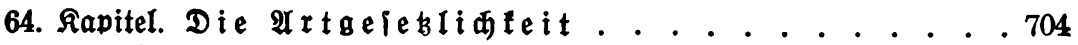

a. Stellung ber „beionderen Geieke“ . . . . . . . . . . . 704

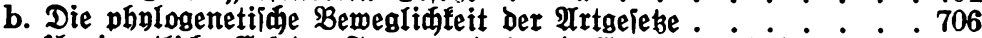

c. Uneigentlide Gelebe. Formmanbel und Gejebeswandel . . . 707 